Heugten, C.M. van, Dekker, J., Deelman, B.G., Dijk, A.J. van, Stehmann-Saris, J.C., Kinebanian, A. Outcome of strategy training in stroke patients with apraxia: a phase II study. Clinical Rehabilitation: 1998, 12(4), 294-303

\begin{tabular}{|l|l|}
\hline $\begin{array}{l}\text { Postprint } \\
\text { Version }\end{array}$ & 1.0 \\
\hline $\begin{array}{l}\text { Journal website } \\
\text { Pubmed link }\end{array}$ & $\underline{\text { http://cre.sagepub.com/cgi/content/abstract/12/4/294 }}$ \\
\hline DOI & $\underline{\mathrm{http} / / \text { www.ncbi.nlm.nih.gov/pubmed/9744665 }}$ \\
\hline
\end{tabular}

This is a NIVEL certified Post Print, more info at http://www.nivel.eu

\title{
Outcome of strategy training in stroke patients with apraxia: a phase II study
}

CM VAn HeUGTEN Netherlands Institute of Primary Health Care and the Institute for Rehabilitation Research, J DEKKER Netherlands Institute of Primary Health Care and the Vrije Universiteit Amsterdam, BG DEELMAN University of Groningen, AJ VAN DIJK Het Roessingh, Center for Rehabilitation, JC STEHMANN-SARIS and A KINEBANIAN School for Occupational Therapy, The Netherlands

Address for correspondence: Caroline M van Heugten, IRV (Institute for Rehabilitation Research), PO Box 192, 6430 AD Hoensbroek, The Netherlands.

Objective: Evaluation of a therapy programme for stroke patients with apraxia. The programme is based on teaching patients strategies to compensate for the presence of apraxia. This programme was designed for assessment and treatment by occupational therapists.

Design: The outcome was studied in a pre-post test design. Measurements were conducted at baseline and 12 weeks later.

Subjects: Thirty-three stroke patients with apraxia were treated at occupational therapy departments in general hospitals, rehabilitation centres and nursing homes.

Main outcome measures: The following measurements were conducted: an apraxia test, a motor functioning test, observation of activities of daily living (ADL), Barthel Index, and an ADL questionnaire for the therapist and the patient.

Results: The patients showed large improvements in ADL functioning on all measures and small improvements on the apraxia test and the motor functioning test. The effect sizes for the disabilities, ranging from 0.92 to 1.06 , were large compared to the effect sizes for apraxia (0.34) and motor functioning (0.19). The significant effect of treatment is also seen when individual improvement and subjective improvement are considered. Measured with the Barthel Index for instance, $71 \%$ of the patients improved.

Conclusions: These results suggest that the programme seems to be successful in teaching patients compensatory strategies that enable them to function more independently, despite the lasting presence of apraxia.

\section{INTRODUCTION}

The term apraxia is widely used to describe one of the more disabling deficits following stroke. It is a disorder affecting the purposeful execution of learned and meaningful activities in which the inability to perform activities is not the result of primary motor or sensory impairments, or of deficits of comprehension, memory or motivation. ${ }^{1,2}$ The patient for instance has sufficient muscle strength and/or motor co-ordination to perform the task. Additionally, the patient does not experience problems because a command was given which was not understood or remembered. Other impairments, like hemiplegia or aphasia, may well be present in the stroke patient, but these deficits are not the cause of the inability to perform 
Heugten, C.M. van, Dekker, J., Deelman, B.G., Dijk, A.J. van, Stehmann-Saris, J.C., Kinebanian, A. Outcome of strategy training in stroke patients with apraxia: a phase II study. Clinical Rehabilitation: 1998, 12(4), 294-303

purposeful acts. The problems in activities of daily living (ADL) functioning are the result of the absence or disturbance in the plan of action. The action plan cannot be retrieved from memory or the patient is unable to properly implement the plan of action to result in a purposeful activity. ${ }^{1,3}$ As De Renzi describes ${ }^{1}$ : a patient with apraxia either does not know what to do because the concept or idea of the motor act is lacking, or does know what to do but not how to do it, because the implementation into a precise motor programme is disrupted.

Despite the disabling effect of apraxia on daily functioning, no studies have been published that evaluate treatment of stroke patients with apraxia. In a meta-analysis of stroke rehabilitation such studies were absent. ${ }^{4}$ A recent review of the empirical literature on apraxia includes definitions, taxonomies and examination procedures, but rehabilitation or therapeutic possibilities are not discussed. ${ }^{5}$ Occasionally, the results of a single case of rehabilitation are found in the literature, ${ }^{6}$ but to our knowledge systematic research into the outcome of apraxia treatment has not been conducted.

In the present study a therapy programme for patients with apraxia was developed. The programme was designed for assessment and treatment of patients with apraxia by occupational therapists. Treatment is aimed at improving the performance of the apractic patients by teaching them strategies. The potential benefit of this is that it enables them to function more independently, despite the persisting presence of apraxia following a stroke. Strategy training is not aimed at recovery of functions. Instead, it aims to improve functioning in spite of the presence of impairments. Compensatory techniques can help the patient to use residual skills effectively and so to minimize the extent to which the impairment influences the performance in daily life. ${ }^{7,8}$

In apraxia, strategy training concentrates on teaching the patient ways to compensate for the impairment. The performance or the environment can be changed or restructured in order to minimize the influence of the distorted functions. Compensation is established by learning to carry out ADL activities using methods different from those the patient was used to prior to the stroke. Compensation can be external or internal. External compensation means that the ADL activity is carried out with help from outside the individual. One speaks of external compensation when, for instance, (technical) aids are used to overcome a disrupted function. When a patient cannot structure the performance due to sequencing problems, the distinct steps in the activity can be presented to the patient by showing him or her pictures. Internal compensation involves cognitive functions other than the distorted ones, for instance visual or verbal functions. An example of internal compensation is teaching the patients consciously to verbalize the proper sequence of distinct steps of which an activity consists, while performing the activity. ${ }^{9-11}$

In this paper the outcome of the therapy programme is evaluated. A group of stroke patients with apraxia was treated according to the guidelines of the programme. Changes were expected in the performance of ADL activities of the patients after treatment. More specifically, improvements in ADL functioning were expected but no or only small changes in the severity of apraxia. These expectations are based on the fact that the therapy programme focuses on teaching patients strategies to compensate for a probably lasting apraxia. There is no specific literature citing that apraxia is irreversible, but by now it is widely acknowledged that it is not plausible to restore higher cognitive functions comp letely. ${ }^{10,11}$ Recovery of apraxia is therefore not a realistic goal for therapy, neither by aiming for spontaneous recovery, nor by repeatedly stimulating brain structures through cognitive retraining.

The present study was exploratory, only one group of patients was tested. This noncontrolled phase II study is essential, however, since it is a necessary first step to be taken before a randomized controlled trial can be relevant. In a phase II study it is possible to investigate whether the intervention or therapy shows positive results. In addition, the assessment instruments and the criteria for selection can be optimized, while the next step should be to formally test these interventions in a clinical trial (phase III research). 
Heugten, C.M. van, Dekker, J., Deelman, B.G., Dijk, A.J. van, Stehmann-Saris, J.C., Kinebanian, A. Outcome of strategy training in stroke patients with apraxia: a phase II study. Clinical Rehabilitation: 1998, 12(4), 294-303

\section{METHODS}

\section{Selection of patients}

Occupational therapists $(\mathrm{n}=26)$ at 16 occupational therapy $(\mathrm{OT})$ departments in general hospitals, rehabilitation centres and nursing homes selected the patients. Patients were eligible if they met the following inclusion criteria: patients diagnosed as having had a stroke in the left hemisphere and as having apraxia, diagnosed by either the referring physician or by the OT on the basis of clinical evaluation. A left-hemisphere stroke is diagnosed when acute clinical symptoms of a focal dysfunction of the left hemisphere are present; these signs and symptoms last at least 24 hours, and probably have a vascular origin only. ${ }^{12,13}$ Apraxia is diagnosed when the patient is fully or partly unable to carry out purposeful activities, this not being due to primary motor or sensory impairments, nor to deficits of comprehension, memory or motivation. Other impairments may be present but they are not the cause of the inability to perform purposeful acts. ${ }^{2}$

Exclusion criteria were: age younger than 25 years or older than 95 years; no working knowledge of the Dutch language; and a set of premorbid and present pathologies: psychiatric or psychogeriatric history, addiction to alcohol, medical or other drugs, contusio cerebri, personality, intellectual or learning disorders, temporary loss of consciousness, or other injuries of the central nervous system. All patients entering the study gave their informed consent and were subsequently treated according to the guidelines of the therapy programme.

\section{Treatment}

The programme for assessment and treatment of apraxia in stroke patients was developed on the basis of an extensive literature search and consultation of experts in the fields of occupational therapy, neurology, neuropsychology and rehabilitation medicine. The patients were treated for a period of 12 weeks. The number of treatments per week was determined by the therapists. In some of the participating institutions the frequency of treatment was determined by institutional policies. Therapists were encouraged to practise with the patients as frequently as possible. On the basis of interviews in clinical prac- tice it appeared that occupational therapists in The Netherlands treat stroke patients about 3-5 times per week, while each treatment session takes 30 min (interviews not published).

During the treatment period activities were trained that were relevant for the patients to (re)learn; the focus of the programme is on disabilities resulting from apraxia that appear in everyday life. The decision about the activity to be trained was made together with the patient. The occupational therapist was encouraged to use a decision tree to guide the choice of an activity or to use a checklist with activities which were carried out by the patient before the occurrence of the stroke and activities which are important for the patient to carry out in the near future.Every two weeks an activity was chosen. After this two-week period a new treatment goal was considered, based upon the improvement in the patient's functioning.

The specific interventions administered during treatment corresponded with the specific problems that were assessed during standardized ADL observations (see section below). ADL activities are conceptualized as being composed of three successive events, according to the framework of information processing: phases of initiation, execution and control. The proper plan of action as well as the correct objects have to be selected (initiation of an activity), followed by adequate performance of the plan (executing the activity), which has to be evaluated in terms of the result (controlling and if necessary correcting the activity). A patient with apraxia who, for example, cannot use objects appropriately, may have a deficit at any one of the stages of which an activity consists. By assessing the different aspects of 
Heugten, C.M. van, Dekker, J., Deelman, B.G., Dijk, A.J. van, Stehmann-Saris, J.C., Kinebanian, A. Outcome of strategy training in stroke patients with apraxia: a phase II study. Clinical Rehabilitation: 1998, 12(4), 294-303

the activity, the nature of the defic i t can be identified and plans for treatment can be formulated accordingly. When a patient predominantly showed problems with initiating an activity, emphasis during treatment was placed upon instructions. The instructions were varied depending on the patient's level of functioning. This means that verbal instructions were given when minimal problems occurred. If, however, the patient could not initiate the activity, the therapist could, for instance, hand the objects to the patient one at a time. Specific assistance was given when the execution of the activity caused problems. Assistance could be verbal or physical. Finally, the therapist offered feedback when the patient did not detect or correct performance errors. Feedback could, for instance, be verbal by telling the patient what went wrong, or the therapist could use a mirror to show to the patient the result. All forms of intervention (instructions, assistance and feedback) could be varied depending on the patient's functioning. ${ }^{14}$ The specific interventions in the form of instructions, assistance and feedback, were presented to the occupational therapist in a protocol. In the protocol the interventions are ordered hierarchically in terms of the level of functioning of the patient. The interventions are presented in the Appendix.

\section{Study design and measurements}

The outcome of the therapy programme was studied using a pre-post test design. The measurements were performed at baseline (pre-test) and 12 weeks later (post-test). On both occasions the researcher visited the patient to administer a set of neuropsychological tests. These tests were conducted in a 1-hour session while the patient sat facing the experimenter in a quiet room suitable for testing. The set of tests contained measures intended to characterize the patient sample, and two outcome measures intended to assess the level of motor functioning and the degree of apraxia. A test of motor functioning was used, consisting of eight tasks concerning the body side opposite to the brain lesion. The eight tasks involve: trunk balance, shoulder movement, arm movement, grasp and release a cylinder, grasp and release a dice, and a test for the sensitivity of the back of the hand. For each task a score of 2 was given when the patient was able to execute it, 1 when the patient could carry it out with some effort, and 0 when the patient was not able to execute it. This way a maximum score of 16 could be reached.

In addition, a test of apraxia was developed, consisting of two subtests, adapted from De Renzi. The first subtest was designed to evaluate the use of objects. Three sets of three objects were presented in different conditions: presentation of the object only by verbal command, without the object present; visual presentation of the object, accompanied by verbal command; and the third condition based on actual object use. A total score of 54 could be reached. The second subtest involved the assessment of the ability to imitate gestures. Six gestures had to be imitated by the patient, immediately after demonstration by the researcher. For this part a score of 36 could be reached. The total score could add up to 90 .

The occupational therapist assessed the level of disabilities in ADL activities. Three outcome measures were selected. First, the occupational therapist executed standardized ADL observations. A procedure was used to guide the OT through specific observations of performance in apractic patients. Before the study started the OTs attended training sessions, during which the use of the observational scales was explained. A videotape of a patient with disabilities as a result of apraxia was shown and scoring was practised. Applying the ADL observations, the OT observed the patient executing four ADL activities, three of which were prescribed: personal hygiene (washing the face and upper body), dressing (putting on a shirt), and preparing food (preparing and eating a sandwich). The fourth activity could be chosen by the OT and depended on the capabilities and needs of the patient or on the department's standard observation procedures. In most cases $(n=30 ; 90 \%)$ this fourth activity turned out to be preparing coffee or tea. These observations were scored on four aspects: independence, initiation, execution and control. Subsequently, the four measures 
Heugten, C.M. van, Dekker, J., Deelman, B.G., Dijk, A.J. van, Stehmann-Saris, J.C., Kinebanian, A. Outcome of strategy training in stroke patients with apraxia: a phase II study. Clinical Rehabilitation: 1998, 12(4), 294-303

were added to arrive at a total score. Next, the Barthel Index was used, which offers a simple and quick, clinically relevant way of both identifying the most important physical disabilities and measuring their overall extent. ${ }^{15-17}$ Finally, the OT was asked to give grades ranging from 1 to 10 for 16 different ADL activities, i.e. the ADL questionnaire (OT). A grade of 1 was given when the performance was very difficult or impossible for the patient; a grade of 10 was given when the performance was perceived as being normal and adequate. The score 'not applicable' was given when the patient had not performed the activity at all since the occurrence of the stroke. The same questionnaire was also presented to the patient (ADL questionnaire (pt)); after the treatment period the patient was asked to judge whether performing these 16 activities had improved. This judgement was represented on a six-point scale ranging from 'completely recovered' to 'much worsened'. The ADL questionnaire is derived from the Rivermead ADL index..$^{18,19}$

\section{Statistical analyses}

The differences between baseline and posttreatment scores were calculated to establish the effect of treatment The t-test for paired samples was used for comparison of measures before and after treatment. Effect sizes were calculated by dividing the mean changes in scores by the standard deviation of the pretest score. An effect size of 0.2 is regarded as small; an effect size of 0.5 as medium; and an effect size of 0.8 or higher indicates a large effect ${ }^{20} \mathrm{~A}$ multivariate analysis of variance (MANCOVA for repeated measures) was executed to test the change in ADL functioning (i.e. disabilities) while correcting for the change in apraxia and motor functioning. To correct for 'spontaneous recovery', the MANCOVAs also included time since stroke as covariate. This way MANCOVAs were executed for the three disability measures separately (i.e. ADL observations, Barthel Index and ADL questionnaire (OT)), taking into account three covariates (i.e. motor functioning, apraxis and time since stroke). Thus, in the repeated measures analysis the disability measures were entered as several dependent variables representing measurements at different times on the same subject. Each of the variables is regarded as a different level of a within-subjects factor. The analyses were at two levels (pre- and posttreatment scores) with three covariates, while the full factorial model is specified and the user miss- ing values are included.

For determining the number of patients who had improved after treatment, the reliable change index (RC) is used. The RC is calculated by $\mathrm{RC}=\left(\mathrm{x}_{2}-\mathrm{x}_{1}\right) / \mathrm{S}_{\text {diff }}$ in which $\mathrm{x}_{1}$ is the score at baseline, $\mathrm{x}_{2}$ is the posttreatment score, and $\mathrm{S}_{\text {diff }}$ is the standard error of the difference between the two scores. $\mathrm{S}_{\text {diff }}$ can also be calculated as $\sqrt{ }\left(2(\mathrm{SE})^{2}\right)$, in which SE is the standard error of measurement. ${ }^{21-23}$ All statistical analyses were carried out using SPSS/PC+, version 5.0. The level of significance was set at 0.01 .

\section{RESULTS}

\section{Patients}

Between December 1993 and February 1995 a total of 45 patients was included in the study. The patient flow is presented in Table 1. For 33 patients pre- and post-treatment scores were available. Twelve patients were excluded from the measurements at 12 weeks for various reasons: comorbidity, right hemisphere stroke, refusal of treatment, death and early discharge. In some cases the set of outcome measures was not complete due to organizational problems within the participating institutions or the current health status of the patient. For this reason the number of patients for which a comparison between baseline and post-treatment could be made is presented in each table. Characteristics of the patients are shown in Table 2. Concerning the size and site of the lesion, detailed information was not gathered systematically, but in most cases clinicians reported that the lesion was located in the parietal area. 
Heugten, C.M. van, Dekker, J., Deelman, B.G., Dijk, A.J. van, Stehmann-Saris, J.C., Kinebanian, A. Outcome of strategy training in stroke patients with apraxia: a phase II study. Clinical Rehabilitation: 1998, 12(4), 294-303

\section{[TABLE 1]}

[TABLE 2]

\section{Outcome}

Table 3 shows the baseline and 12-week results of the outcome measures. Improvement in functioning was seen in most outcome measures. The change in functioning after treatment was significant for the test of motor functioning $(\mathrm{t}(33)=3.28, \mathrm{p}<0.05)$ and for the test of apraxia $(\mathrm{t}(31)=3.28, \mathrm{p}<0.01)$.

The disability measures (i.e. ADL observations, Barthel Index and ADL questionnaire (OT) showed strong and significant results. The ADL observations showed a reduction of disabilities $(\mathrm{t}(25)=6.84 ; \mathrm{p}<0.00)$. This result is also seen in the Barthel Index $(\mathrm{t}(28)=5.99$; $\mathrm{p}<0.01)$ and the ADL questionnaire (OT) $(\mathrm{t}(28)=6.47 ; \mathrm{p}<0.01)$.

\section{[TABLE 3]}

\section{Effect size}

As was expected, the effect sizes for the apraxia and motor functioning were small compared to the effect sizes for the disabilities (Table 3). The effect size statistic of the ADL questionnaire was highest (1.06) and the effect size of the test of motor functioning was lowest (0.19).

\section{Multivariate analyses}

MANCOVAs for repeated measures were executed to test whether the improvement in ADL functioning was significant when corrected for changes in apraxia and motor functioning, and the time since stroke. These analyses were carried out separately for the ADL observations, the Barthel Index, and the ADL questionnaire. The ADL observations showed a significant improvement in functioning when the three covariates were taken into account $(F(1,20)=38.65 ; p<0.01)$. Similar results were obtained for the Barthel Index and the ADL questionnaire; the analyses of variance showed a significant improvement after treatment $(\mathrm{F}(1,22)=23.16, \mathrm{p}<0.01)$ and $\mathrm{F}(1,22)=18.87, \mathrm{p}<0.01$ respectively $)$.

\section{Individual changes}

In clinical practice it is interesting to know whether a group of patients shows significant improvement as a result of treatment but, perhaps more importantly, whether an individual patient responds positively to the treatment given .

For each patient the reliable change index (RC) was calculated. Based on the RC, significantly improved patients can be defined as patients showing an RC higher than 1.96, because in this case significant change $(p<0.05)$ occurs (see Methods section). Table 4 presents the percentage of patients showing significant improve- ment from baseline to 12 weeks follow-up. As was hoped for, on an individual level most patients showed significant improvement in ADL functioning (ADL observations, Barthel Index and ADL questionnaire). Improvement in motor functioning and apraxia occurred in a minority.

[TABLE 4] 
Heugten, C.M. van, Dekker, J., Deelman, B.G., Dijk, A.J. van, Stehmann-Saris, J.C., Kinebanian, A. Outcome of strategy training in stroke patients with apraxia: a phase II study. Clinical Rehabilitation: 1998, 12(4), 294-303

\section{Subjective improvement}

In addition to objective measures of improvement, it is important to know how the patients themselves evaluate the effects of treatment. In our study the perceived treatment effect was determined by means of the ADL questionnaire (pt). The results on this six-point scale are shown in Table 5. As the table shows, $84 \%$ of the patients $(n=22)$ judged the treatment to have resulted in complete recovery or much improvement. Comparison of this result with the ADL questionnaire completed by the OT before and after treatment showed that in $87 \%$ of the cases the patient and the therapist agreed that improvement in functioning had taken place (not shown in a table).

\section{[TABLE 5]}

\section{DISCUSSION}

To our knowledge this is the first study on the outcome of treatment of stroke patients with apraxia. The treatment was offered by means of a therapy programme aiming at teaching patients strategies to compensate for the apraxia. It was expected that improvement in ADL functioning would be more evident than recovery of the apraxia and/or motor impairments.

The expectations were confirmed. The results of this study showed large and significant effects on all measures in ADL functioning. The improvement in functioning was found in the ADL observations, as well as the Barthel Index and the ADL questionnaire. There was significant, but small change on the test of apraxia and the test of motor functioning. The effect sizes of these measures were small. The results were further supported when changes in individual functioning and subjective improvement were considered.

Similar results were obtained in a study of memory rehabilitation. ${ }^{7,10}$ Patients with memory problems were given strategy training, as opposed to drill and practise or no treatment. The group of patients receiving the strategy training showed sign i ficantly improved subjective as well as objective memory performance, but no significant improvement was found with respect to memory tasks for which the use of strategies was not possible. The more recent studies in cognitive rehabilitation thus suggest that strategy training can be applied when spontaneous recovery seems unlikely since the impairment is frequently irreversible or the time since injury is prominent.

We should be somewhat modest concerning the successful treatment outcome. Since the design was a pre-experimental one (i.e. no control group was present), the conclusions remain tentative. In the current design spontaneous recovery is not controlled for. However, the time post stroke was more than two months on average, which exceeds the acute phase for recovery. More importantly, the improvement in ADL functioning was still sign i ficant when corrected for the improvement on the test of apraxia, the test of motor functioning, and the time since stroke. Thus, the multivariate analyses support the conclusion that strong improvement in ADL functioning was found after treatment. Correction for confounding factors did not alter this robust outcome. And finally, if the improvements were the result of spontaneous recovery, one would expect a comparable effect on all measures. The differences between the magnitude of the effect sizes for the measurements allow the conclusion that improvement in ADL functioning was considerable, while recovery of the underlying impairments (apraxia and motor functioning) was less marked. Testing of our conclusions in a controlled study remains a logical and necessary next step.

The number of treatments per week varied between the patients. Categorizing the patients according to the amount of treatment received is not possible on the basis of the data. Analyses determining whether degree of improvement was related to amount of training were thus not performed. 
Heugten, C.M. van, Dekker, J., Deelman, B.G., Dijk, A.J. van, Stehmann-Saris, J.C., Kinebanian, A. Outcome of strategy training in stroke patients with apraxia: a phase II study. Clinical Rehabilitation: 1998, 12(4), 294-303

Because of the assumed effect of care and attention given, and because of general motivational aspects, it is not possible to attribute the positive effect of treatment solely to the strategy training. This issue cannot be resolved given the current research design. However, one would expect care and attention to influence all measures equally, which is not the case.

It is therefore concluded that the therapy programme succeeded in teaching patients compensatory strategies, which enabled them to function more independently. Recovery of the apraxia or motor impairments is a less likely explanation for the successful outcome.

\section{Acknowledgements}

This study was supported by a grant to J Dekker from de Nationale Commissie Chronisch Zieken (the National Committee for Chronically Ill People).

\section{REFERENCES}

1 Renzi E de. Apraxia. In: Boller F, Grafman J eds. Handbook of neuro-psychology, volume 2. Amsterdam: Elseviers Science, 1989.

2 Kolb B, Whishaw IQ. Fundamentals of human neuropsychology, third edition. New York: Freeman, 1990.

3 Renzi E de, Lucchelli F. Ideational apraxia. Brain 1988; 111: 1173-85.

4 Ottenbacher $\mathrm{KJ}$, Jannell S. The results of clinical trials in stroke rehabilitation research. Arch Neurol 1993; 50: 37-44.

5 Tate RL, McDonald S. What is apraxia? The clinician's dilemma. Neuropsychol Rehabil 1995; 5(4): 273-97.

6 Pilgrim E, Humphreys GW. Rehabilitation of a case of ideomotor apraxia. In: Riddoch MJ, Humphreys GW eds. Cognitive neuropsychology and cognitive rehabilitation. Hove: Lawrence Erlbaum Associates, 1994.

7 Berg IJ, Koning-Haanstra M, Deelman BG. Longterm effects of memory rehabilitation: A controlled study. Neuropsychol Rehabil 1991; 1: 97-111.

8 Randomski MV. Cognitive rehabilitation: Advancing the stature of occupational therapy. Am J Occup Ther 1994; 48: 271-73.

9 Prigatano GP. Recovery and cognitive retraining after craniocerebral trauma. J Learning Disabil 1987; 20: 603-13.

10 Berg IJ. Memory rehabilitation for closed head injured patients [dissertation]. University of Groningen, Groningen, 1993.

11 Zomeren AH van, Brouwer WH. Clinical neuropsychology of attention. Oxford: Oxford University Press, 1994.

12 World Health Organization, Special Report. Stroke-1989. Recommendations on stroke prevention, diagnosis, and therapy. Stroke 1989; 20: 1407-31.

$13 \mathrm{Crevel} \mathrm{H}$ van. Consensus cerebrovascular accident. Nederlands Tijdschrift voor Geneeskunde 1991; 135 2280-88. 
Heugten, C.M. van, Dekker, J., Deelman, B.G., Dijk, A.J. van, Stehmann-Saris, J.C., Kinebanian, A. Outcome of strategy training in stroke patients with apraxia: a phase II study. Clinical Rehabilitation: 1998, 12(4), 294-303

14 Stehmann-Saris JC, Heugten CM van, Kinebanian A, Dekker J. Ergotherapie protocol voor diagnostieken behandeling van apraxie bij CVA-patienten. Utrecht: NIVEL, Amsterdam: HVA, 1996.

15 Wade DT. Measurement in neurological rehabilitation. Oxford: Oxford University Press, 1992.

16 Collin C, Wade DT, Davies S, Horne V. The Barthel ADL index: a reliability study. Int Disabil Stud 1988; 10: 61-63.

17 Wade DT, Collin C. The Barthel ADL index: a standard measure of physical disability? Int Disabil Stud 1988; 10: 64-67.

18 Whiting S, Lincoln NB. An ADL assessment for stroke patients. Br J Occup Ther 1980; 43: 44-46.

19 Lincoln NB, Edmans JA. A re-validation of the Rivermead ADL-scale for elderly patients with stroke. Age Ageing 1990; 19: 9-24.

20 Cohen J. Statistical power analysis for the behavioural sciences. London: Academic Press, 1977.

21 Jacobson NS, Follette WC, Revensdorf D, Baucom DH, Hahlweg K, Margolin G. Variability in outcome and clinical significance of behavioural marital therapy: a reanalysis of outcome data. J Consult Clin Psychol 1984; 52: 497-504.

22 Christensen L, Mendoza JL. A method of assessing change in a single subject: An alteration of the RC index. Behaviour Ther 1986; 17: 305-308.

23 Jacobson NS, Truax P. Clinical significance: A statistical approach to defining meaningful change in psychotherapy research. J Consult Clin Psychol 1991 ; 59: 12-19.

\section{TABLES}

Table 1 Patient flow

\begin{tabular}{|c|c|}
\hline \\
\hline $\begin{array}{l}\text { Regiscered patients }(n=48) \\
\text { Patients included at baseline }(n=45)\end{array}$ & $\begin{array}{l}\text { Three patients excluded: } \\
\text { comorbidity }(n=1) \\
\text { right-hemisphere stroke }(n=2)\end{array}$ \\
\hline Patients receiving treatment $(n=43$ ) & $\begin{array}{l}\text { Two patients without treatment: } \\
\text { patient refused treatment }(n=2)\end{array}$ \\
\hline Post-treatment measures at 12 weeks $(n=33)$ & $\begin{array}{l}\text { Ten patients received no post-treatment measures: } \\
\text { patient died }(n=2) \\
\text { early discharge }(n=8)\end{array}$ \\
\hline
\end{tabular}


Heugten, C.M. van, Dekker, J., Deelman, B.G., Dijk, A.J. van, Stehmann-Saris, J.C., Kinebanian, A. Outcome of strategy training in stroke patients with apraxia: a phase II study. Clinical Rehabilitation: 1998, 12(4), 294-303

Table 2 Characteristics of the patients $(n=33)$

\begin{tabular}{ll}
\hline Gender & 18 \\
Male & 15 \\
Female & \\
Age (years) & 70.1 \\
$\quad$ Mean & 11.0 \\
SD & $39-91$ \\
Range & \\
Institution & 11 \\
$\quad$ Hospital & 14 \\
Rehabilitation centre & 8 \\
$\quad$ Nursing home & \\
Cva & 9 \\
$\quad$ Cerebral haemorrhage & 23 \\
Cerebral infarction & 1 \\
Unknown & \\
Time since CVA (weeks) & \\
Mean & 8.9 \\
SD & 5.2 \\
Range & $1.6-21.4$ \\
\hline
\end{tabular}

Table 3 Treatment outcome

\begin{tabular}{lcrrrr}
\hline Instruments & $\mathrm{n}$ & $\begin{array}{l}\text { Baseline } \\
\text { mean (SD) }\end{array}$ & $\begin{array}{l}\text { Post-treatment } \\
\text { mean (SD) }\end{array}$ & $\begin{array}{c}\text { p-value } \\
\text { Effect } \\
\text { size }\end{array}$ \\
\hline Motor functioning & 33 & $7.64(6.2)$ & $8.80(6.3)^{*}$ & 0.03 & 0.19 \\
Apraxia & 31 & $58.10(28.0)$ & $67.58(26.9)^{* *}$ & $<0.001$ & 0.34 \\
ADL observations & 25 & $0.98(0.65)$ & $0.38(0.47)^{* *}$ & $<0.001$ & 0.92 \\
Barthel Index & 28 & $10.14(5.54)$ & $14.89(5.04)^{* *}$ & $<0.001$ & 0.86 \\
ADL questionnaire (OT) & 28 & $5.36(1.57)$ & $7.03(1.79)^{* *}$ & $<0.001$ & 1.06 \\
\hline
\end{tabular}

$* p<0.01, * p<0.05$

Table 4 Proportions and percentages of patients improving significantly

\begin{tabular}{lcc}
\hline Outcome measures & Proportion & $\%$ \\
\hline Motor functioning & $7 / 33$ & 21 \\
Apraxia & $11 / 31$ & 35 \\
ADL observations & $20 / 25$ & 80 \\
Barthel Index & $20 / 28$ & 71 \\
ADL questionnaire (OT) & $21 / 28$ & 75 \\
\hline
\end{tabular}

Table 5 Subjective improvement

\begin{tabular}{lrr}
\hline Judgement & $\mathrm{n}$ & $\%$ \\
\hline Completely recovered & 7 & 27 \\
Much improved & 15 & 57 \\
Slightly improved & 2 & 8 \\
No change & 1 & 4 \\
Slightly worsened & 1 & 4 \\
Much worsened & 0 & 0 \\
\hline
\end{tabular}


Heugten, C.M. van, Dekker, J., Deelman, B.G., Dijk, A.J. van, Stehmann-Saris, J.C., Kinebanian, A. Outcome of strategy training in stroke patients with apraxia: a phase II study. Clinical

\section{APPENDIX - INTERVENTIONS}

The specific interventions are built up in a hierarchical order, depending upon the level of functioning of the patient. The therapist can use instructions, assistance, and feedback.

\section{Instructions}

The occupational therapist can give the following instructions:

- Start with a verbal instruction.

- Shift to a relevant environment for the task at hand.

- Alert the patient:

by touching.

using the patient's name,

asking questions about the instruction.

- Use gestures, point to the objects.

- Demonstrate (part of) the task.

- Show pictures of the activity.

- Write down the instruction.

- Place the objects near the patient, point to the objects, put the objects in the proper sequence.

- Hand the objects one at a time to the patient.

- Start the activity together with patient one or more times.

- Adjust the task to make it easier for the patient.

- Finally, take over the task because all efforts did not lead to the desired result.

\section{Assistance}

The following forms of assistance can be given by the therapist:

- There is no need to assist the patient during the execution of the activity.

- Verbal assistance is needed:

by offering rhythm and not interrupting performance,

to stimulate verbalization of the steps in the activity,

to name the steps in the activity or name the objects,

to direct the attention to the task at hand.

- Use gestures, mimics, and vary intonation in your speech.

- Show pictures of the proper sequence of steps in the activity.

- Physical assistance is needed: by guiding the limbs,

in positioning the limbs,

to use the neurodevelopmental treatment method (NDT),

to use aids to support the activity,

to taking over until the patient starts performing,

to provoke movements.

- Finally, take over the task.

\section{Feedback}

Feedback can be offered in the following ways:

- No feedback is necessary because the result is adequate.

- Verbal feedback is needed in terms of the result (knowledge of results).

- Verbal feedback by telling the patient to consciously use the senses to evaluate the result (tell the patient see, hear, feel, smell or taste).

- Physical feedback is needed in terms of the result (knowledge of results): to evaluate the posture of the patient,

to evaluate the position of the limbs, to support the limbs.

- Physical feedback is given by pointing or handing the objects to the patient.

- Verbal feedback is needed in terms of performance (knowledge of performance).

- Physical feedback is needed in terms of performance (knowledge of performance).

- Place the patient in front of a mirror.

- Make video recordings of the patient's performance and show the recordings.

- Take over the control over the task and correct possible errors. 